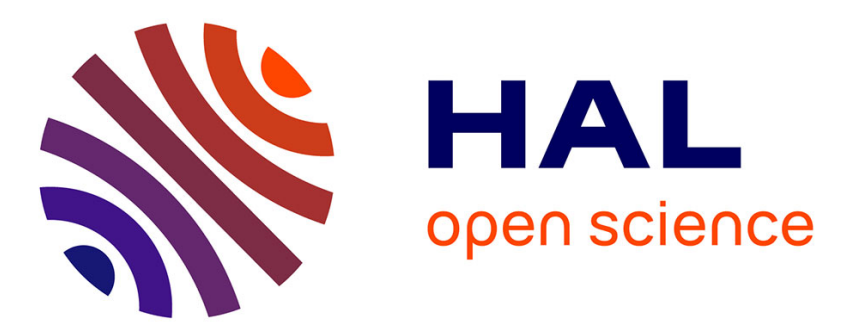

\title{
Joint Optimization of Radio Resources in Small and Macro Cell Networks
}

Chung Shue Chen, François Baccelli, Roullet Laurent

\section{To cite this version:}

Chung Shue Chen, François Baccelli, Roullet Laurent. Joint Optimization of Radio Resources in Small and Macro Cell Networks. IEEE Vehicular Technology Conference, IEEE, May 2011, Budapest, Hungary. 10.1109/VETECS.2011.5956746 . hal-00745117

\section{HAL Id: hal-00745117 https://hal.inria.fr/hal-00745117}

Submitted on 24 Oct 2012

HAL is a multi-disciplinary open access archive for the deposit and dissemination of scientific research documents, whether they are published or not. The documents may come from teaching and research institutions in France or abroad, or from public or private research centers.
L'archive ouverte pluridisciplinaire HAL, est destinée au dépôt et à la diffusion de documents scientifiques de niveau recherche, publiés ou non, émanant des établissements d'enseignement et de recherche français ou étrangers, des laboratoires publics ou privés. 


\title{
Joint Optimization of Radio Resources in Small and Macro Cell Networks
}

\author{
Chung Shue Chen*, François Baccelli*, and Laurent Roullet ${ }^{\dagger}$ \\ *TREC, INRIA-ENS, 75214 Paris, France \\ $\dagger$ Alcatel-Lucent Bell Labs, 91620 Nozay, France \\ INRIA-Alcatel-Lucent Bell Labs Joint Research Center, France \\ Email: chung-shue.chen@inria.fr, francois.baccelli@ens.fr, laurent.roullet@alcatel-lucent.com
}

\begin{abstract}
We propose and analyze a class of distributed algorithms performing the joint optimization of radio resources in heterogeneous cellular networks made of a juxtaposition of macro and small cells. We show that within this context, it is essential to use algorithms able to simultaneously solve the problems of channel selection, user association and power control. In such networks, the unpredictability of the cell and user patterns also requires self-optimized schemes. The proposed solution is inspired from statistical physics and is based on Gibbs sampler. It can be implemented in a fully distributed way and nevertheless achieves minimal system-wide potential delay. Simulation results show that it outperforms today's default operational methods in both throughput and energy efficiency.
\end{abstract}

\section{INTRODUCTION}

Due to the high demand of broadband services and new applications, wireless networking is facing the challenge of supporting fast increasing data traffic with requirements of full coverage and energy-efficient radio resource allocation [1]. To enhance the network capacity and support pervasive broadband service, reducing cell size is one of the most effective approaches [2]. Deployment of small cell or femtocell base stations (BS) has a great potential to improve the spatial reuse of radio resource and also enhance transmit power efficiency. It is foreseen that the next generation mobile networks will consist of heterogeneous macro and small cells with different capabilities including transmit power and coverage range.

However, in such networks due to the unpredictability of the BS and user patterns, network self-organization and selfoptimization becomes necessary in order to relax the heavy human efforts required by conventional network planning and optimization tasks [3]. Autonomic management and configuration is highly desirable [4]. For example, user association and radio resource allocation such as transmit power and channel selection should be automatically adjusted so as to reduce the system's capital and operational expenditure (CAPEX/OPEX).

The present paper aims at designing distributed algorithms performing self-optimization for future macro and small cell (e.g., LTE and femtocell) mixed networks [5]. In radio resource management, (i) power control, (ii) user association and (iii) channel selection are essential elements. In traditional networks made of macro cells only, optimizing any of the above three elements independently could improve the system performance. However, this may not be true in heterogeneous networks made of a juxtaposition of macro and small cells. We give below two examples to illustrate the problem that may happen when conducting these optimizations separately in both the downlink (DL) and uplink (UL).

Consider the DL scenario in Fig. 1 where there are two mobile users $u$ and $v$ under the macro and small cell BS $a$ and $b$ which have different maximum transmit powers and coverage ranges. Notice that $u$ can be covered by the macro cell BS $a$ but it is located near the edge of $a$ 's coverage. Meanwhile, it is too close to the small cell BS $b$ which will have a strong impact on its received signal-to-interferenceplus-noise-ratio (SINR). Here, transmit power optimization will not be effective without prior user association and channel selection optimization. One may consider the option in which $u$ and $v$ both associate with $b$. However, this may overload $b$. From the viewpoint of load balancing, it is better to have the two users attached to different cells, e.g., $u$ attaches to $a$. However, $u$ will then have low SINR as long as the two transmissions use a same channel. Clearly, one should consider assigning two different channels for these two transmitterreceiver pairs and hence conduct a joint user association and channel selection optimization. If the system involves more users and cells, power control should be conducted as well to mitigate interference. This requires a joint optimization of all the three elements.

Fig. 2 shows a similar problem in the UL. Consider that one first conducts user association optimization. Since $v$ is closer to BS $b$ than to BS $a$, from the viewpoint of load balancing the recommended user association should be as follows: $u$ attaches to $a$ while $v$ attaches to $b$. As $u$ is far away from its $\mathrm{BS} a$, the transmit power has to be high enough. This will however yield a strong interference to the signal received at $b$, which is transmitted from $v$. Note that in this case, user association optimization, power control or even their joint optimization are not able to solve the problem. However, if one considers channel allocation and tries to select two different channels for these two pairs, a joint optimization can resolve the conflict and enhance the overall performance.

To decide which aspects should be optimized and what is the proper optimization sequence if each is to be conducted separately, some guidelines can be derived from careful experimental work and case studies (see e.g., [6] for 802.11 WLAN). Explicit rules can be proposed if the BS and user patterns are known or predictable. However, for situations where their patterns are unpredictable as those with small cells, no simple and universal rule is known and a joint optimization is necessary to achieve the best performance.

The joint optimization of the above three aspects is in 


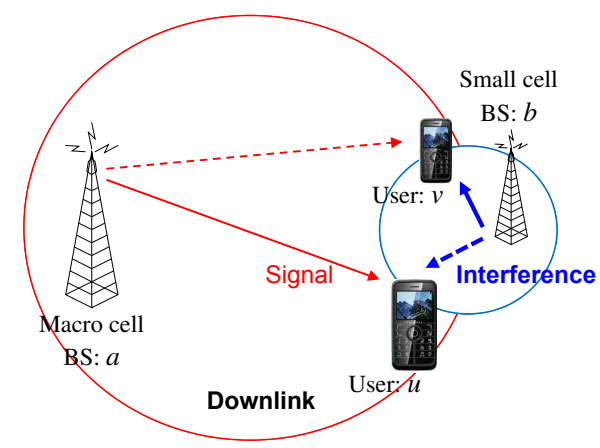

Fig. 1. Since user $u$ is far from its $\operatorname{BS} a$, the received signal at $u$ may suffer strong interference due to the transmission of small cell $\mathrm{BS} b$ destined to $v$.

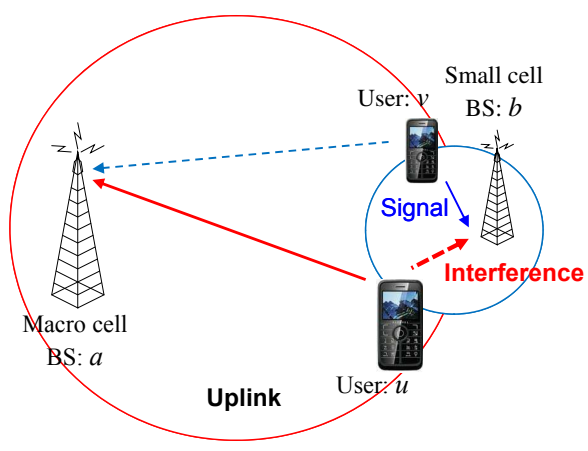

Fig. 2. The signal received at BS $b$ sent from $v$ can be strongly interfered by $u$ 's transmission since $u$ has to use a high power in order to send to $a$.

general a difficult problem even if centralized coordination and global information exchange are allowed. Even in the separate optimization case, the determination of the transmission powers maximizing system throughput is challenging in multiple interfering link case since the problem is in general non-convex. More generally, there is a lack of efficient algorithm operating in a distributed manner and ensuring global optimality in the above joint optimization.

In this paper, we propose and analyze a class of distributed algorithms performing the joint optimization of radio resources in the generalized heterogenous macro and small cell networks. The proposed solution is inspired from statistical physics and is based on Gibbs sampler (see, e.g., [7]). The solution is a generalization of the work in [8] (which only takes into account power control and user association and is thus limited to homogeneous cellular networks).

The paper is organized as follows. Section II describes the system model and problem setup. Section III presents the proposed solution and algorithm. Section IV reports its performance and compares the solution to today's default operation in terms of throughput and power utilization efficiency. Finally, Section $\mathrm{V}$ contains the conclusion.

\section{System ModeL}

We consider a reuse-1 radio system with a set of BS $\mathcal{B}$ serving a population of users denoted by set $\mathcal{U}$. For each user $u \in \mathcal{U}$, it is assumed that there is a pair of orthogonal channels for the UL and DL. Since there is no interference between the
UL and DL, for simplicity here we only consider the DL. However, the discussion can be generalized to the UL as well.

We assume that users can associate with any neighboring BS $b \in \mathcal{B}$ in the network which could be a macro or small cell, which is referred to as open access. Today's default operation attaches each user to the BS with the highest received power [9]. This is however clearly sub-optimal.

Let $\mathcal{C}$ be the set of channels (e.g., frequency band) which are common to all the base stations. The transmit power used by a $\mathrm{BS} b_{u} \in \mathcal{B}$ to send a signal to its user $u$ in a channel $c \in \mathcal{C}$ is denoted by $P_{u}(c)$. Here, for simplicity we consider that a user $u$ will only take one channel, denoted by $c_{u} \in \mathcal{C}$. In general, a user may get served by several orthogonal channels simultaneously. One can consider the case where a user with higher traffic demand may request a multiplicative number of transmissions in parallel. A generalization is possible. Here due to a lack of space we will not go into the detail.

The SINR at $u$ is then expressible as:

$$
\operatorname{SINR}_{u}=\frac{P_{u}(c) l\left(b_{u}, u, c\right)}{N_{u}+\sum_{v \in \mathcal{U}, v \neq u} \alpha(v, u, c) P_{v}(c) l\left(b_{v}, u, c\right)},
$$

where $N_{u}$ denotes the receiver noise at $u, l\left(b_{u}, u, c\right)$ is the signal attenuation from $b_{u}$ to $u$ in channel $c$, and $\alpha(v, u, c)$ represents the orthogonality factor on the transmission destined to $v \in \mathcal{U}$. Note that $0 \leq \alpha(\cdot) \leq 1$. Here, we consider that adjacent channel interference is negligible compared to co-channel interference. One may assume that when $b_{u}=b_{v}$, $\alpha(v, u, c)=\alpha$; otherwise, $\alpha(v, u, c)=\beta$, where $\alpha$ and $\beta$ are constants. In simple case, one may consider $\alpha=\beta=1$.

Under the additive white Gaussian noise (AWGN) model, the achievable data rate at $u$ in $\mathrm{bit} / \mathrm{s} / \mathrm{Hz}$ is given by:

$$
r_{u}=K \log \left(1+\operatorname{SINR}_{u}\right),
$$

where $K$ is a constant depending on the width of the frequency band.

To achieve network throughput enhancement while supporting bandwidth sharing fairness, we adopt the notion of minimal potential delay fairness proposed in [10]. This solution for bandwidth sharing is intermediate between max-min and proportional fairness. It aims at minimizing the system-wide potential delay and is explained below. Instead of maximizing the sum of throughputs, i.e., $\sum r_{u}$, which often leads to very low throughput for some users, we minimize the sum of the inverse of throughput, i.e., $\sum r_{u}^{-1}$, which can be seen as the total delay spent to send an information unit to all the users [11]. A bandwidth allocation that provides minimal potential delay fairness is one that minimizes the following cost function:

$$
C=\sum_{u \in \mathcal{U}} r_{u}^{-1}
$$

where very low throughputs will significantly increase the cost.

For mathematical convenience (see below), we actually minimize the cost function called the global energy in following 
the terminology of Gibbs sampler [7] defined below:

$$
\mathcal{E}=\sum_{u \in \mathcal{U}} \frac{1}{e^{\frac{r_{u}}{K}}-1}=\sum_{u \in \mathcal{U}} \frac{1}{\operatorname{SINR}_{u}}
$$

instead of (3). Note that if one operates in low SINR regime such that the achievable data rate of a user is proportional to its SINR, then minimizing $C$ is equivalent to minimizing $\mathcal{E}$, which is a surrogate of $C$. We see that (3) and (4) have quite similar characteristics. The difference is that $\left(e^{\frac{r_{u}}{K}}-1\right)^{-1}$ increases more significantly than $r_{u}^{-1}$ when $r_{u}$ is low. The overall cost will increase more substantially. Minimizing $\mathcal{E}$ thus favors a higher level of bandwidth sharing fairness.

By (1), the global energy $\mathcal{E}$ in (4) is expressible as:

$$
\mathcal{E}=\sum_{u \in \mathcal{U}} \frac{N_{u}+\sum_{v \in \mathcal{U}, v \neq u} \alpha\left(v, u, c_{u}\right) P_{v}\left(c_{u}\right) l\left(b_{v}, u, c_{u}\right)}{P_{u}\left(c_{u}\right) l\left(b_{u}, u, c_{u}\right)}
$$

which can be re-written as:

$$
\begin{aligned}
\mathcal{E}= & \sum_{u \in \mathcal{U}} \frac{N_{u}}{P_{u}\left(c_{u}\right) l\left(b_{u}, u, c_{u}\right)}+ \\
& \sum_{\{u, v\} \subseteq \mathcal{U}}\left(\frac{P_{v}\left(c_{u}\right) l\left(b_{v}, u, c_{u}\right)}{P_{u}\left(c_{u}\right) l\left(b_{u}, u, c_{u}\right)}+\frac{P_{u}\left(c_{v}\right) l\left(b_{u}, v, c_{v}\right)}{P_{v}\left(c_{v}\right) l\left(b_{v}, v, c_{v}\right)}\right)
\end{aligned}
$$

where and in the coming presentation for notational simplicity we will omit the notation $\alpha(\cdot)$ unless it should be completed.

The optimization problem is then to find a configuration of user association, channel selection and power allocation which minimizes $\mathcal{E}$. It is clear that the problem has a high combinatorial complexity and is in general hard to solve for large networks. However, if the cost function has the form given above, the minimization can be conducted by Gibbs sampler and can be implemented in a fully distributed way. This explains the choice made in (4).

\section{Network SElF-Optimization}

We now describe the network self-optimization scheme. It is based on a Gibbs sampler operating on a graph $\mathcal{G}$ of the network which can be defined as follows:

- The set of nodes in $\mathcal{G}$ is the set of users denoted by $u \in \mathcal{U}$.

- Each node is endowed with a state variable $\mathbf{s}$ belonging to a finite set $\mathcal{S}$. The state of a node is its user association, channel and the transmit power, denoted by $\left(b_{u}, c_{u}, P_{u}\right)$. Here, we consider that transmit power is discretized.

- The set of neighbors of a node in $\mathcal{G}$ is the set of all users $v \neq u$ such that the power of the signal received from $b_{v}$ at $u$ is above a specific threshold, say $\theta$. Notice that $b_{v}$ is then considered as the neighboring base station of $u$.

From (6), we see that $\mathcal{E}$ derives from a potential function [7] $V(\mathcal{V})$ as follows: for all subsets $\mathcal{V} \subseteq \mathcal{U}$, we can have $\mathcal{E}=\sum\{V(\mathcal{V}) \mid \mathcal{V} \subseteq \mathcal{U}\}$, where $V(\cdot)$ has the following form:

$$
\begin{cases}V(\mathcal{V})=\frac{N_{u}}{P_{u}\left(c_{u}\right) l\left(b_{u}, u, c_{u}\right)} & \text { if } \mathcal{V}=\{u\} \\ V(\mathcal{V})=\frac{P_{v}\left(c_{u}\right) l\left(b_{v}, u, c_{u}\right)}{P_{u}\left(c_{u}\right) l\left(b_{u}, u, c_{u}\right)}+\frac{P_{u}\left(c_{v}\right) l\left(b_{u}, v, c_{v}\right)}{P_{v}\left(c_{v}\right) l\left(b_{v}, v, c_{v}\right)} & \text { if } \mathcal{V}=\{u, v\} \\ V(\mathcal{V})=0 & \text { if }|\mathcal{V}| \geq 3\end{cases}
$$

A global energy which derives from the above potential function satisfying the condition $V(\mathcal{V})=0$ for $|\mathcal{V}| \geq 3$ is hence amenable to a distributed optimization using Gibbs sampler with the local energy at each node defined below:

$$
\mathcal{E}_{u}=\sum\{V(\mathcal{V}) \mid u \in \mathcal{V}, \mathcal{V} \subseteq \mathcal{U}\}
$$

which by the above definition can be re-written as:

$$
\mathcal{E}_{u}=\underbrace{\frac{N_{u}+\sum_{v \neq u} P_{v}\left(c_{u}\right) l\left(b_{v}, u, c_{u}\right)}{P_{u}\left(c_{u}\right) l\left(b_{u}, u, c_{u}\right)}}_{=1 /\left(\operatorname{SINR}_{u}\right)}+\sum_{v \neq u} \frac{P_{u}\left(c_{v}\right) l\left(b_{u}, v, c_{v}\right)}{P_{v}\left(c_{v}\right) l\left(b_{v}, v, c_{v}\right)},
$$

where the first term is the "selfish" part of the energy function which is small if $\operatorname{SINR}_{u}$ is large, while the second term is the "altruistic" part which is small if the power of interference incurred by all the other users (i.e., $v \neq u$ ) because of $u$ compared to the power received from their own BS is small.

Note that the above formulation handles joint power, channel, and user association optimization. However, it can be easily adapted to separate optimization and special cases.

In the following, we describe the distributed algorithm based on Gibbs sampler for the network self-optimization.

- Each BS separately triggers a state transition for one of its users picked at random, say $u$, using a local random timer. It samples the random variables $\left(b, c, P_{u}\right)$ and selects a state $\mathbf{s}$ with a probability proportional to $e^{-\mathcal{E}_{u}(\mathbf{s}) / T}$ based on $\mathcal{E}_{u}$, where $T$ is a parameter called the temperature.

- The dynamics based on these local transitions, called the Gibbs sampler, will drive the network to a steady state which is the Gibbs distribution associated with $\mathcal{E}$ and $T$, and has the following distribution in steady state:

$$
\pi_{T}(\mathbf{s}, u \in \mathcal{U})=c \cdot e^{-\mathcal{E}(\mathbf{s}) / T},
$$

with $c$ a normalizing constant.

- This distribution puts more mass on low energy configurations. When $T \rightarrow 0$ in a proper way, the distribution $\pi_{T}(\cdot)$ will converge to a Dirac mass at the optimal state of minimal cost if it is unique.

The above state transition consists in selecting a new state for user $u$ according to the following probability distribution which in fact only depends on the states of its neighbor nodes:

$$
\pi_{u}(\mathbf{s})=\frac{e^{-\mathcal{E}_{u}(\mathbf{s}) / T}}{\sum_{\mathbf{s} \in \mathcal{S}} e^{-\mathcal{E}_{u}(\mathbf{s}) / T}},
$$

where $T>0$ is the temperature and $\mathcal{S}$ refers to the finite set of allowable choices. The Gibbs sampler selects a state of low energy with high probability. As aforementioned, for practical reason, power level is discretized such that $P_{u} \in$ $\left\{0, P_{\delta}, 2 P_{\delta}, \ldots, P_{\max }\right\}$, where $P_{\max }$ is the maximum transmit power and $P_{\delta}$ is the step size. Two BS, say $a$ and $b$, are called implicit neighbors if at least one user associated with one BS receives the signal of the other BS above the threshold.

To determine $\pi_{u}(\mathbf{s})$ for Gibbs sampler, we need to evaluate $\mathcal{E}_{u}(\mathbf{s})$. Some measurement and information exchange between neighboring base stations and users are thus required. Following the explicit definition of $\mathcal{E}_{u}$ under (7), we consider that for 
the evaluation of the first term in $\mathcal{E}_{u}$, a user $u$ will estimate the following data and report to its base station $b_{u}$ :

1) the receiver noise: $N_{u}$,

2) the sum of received interferences in each channel $c \in \mathcal{C}$ to be selected: $\sum_{v \neq u} P_{v}(c) l\left(b_{v}, u, c\right)$, and

3) the path loss: $l(b, u, c)$, for each $c$ and for each of its neighboring $\mathrm{BS} b$ to be selected.

For the evaluation of the second term, a user $v$ will estimate the following information and report to its base station $b_{v}$ :

1) the power of its received signal: $P_{v}\left(c_{v}\right) l\left(b_{v}, v, c_{v}\right)$, and

2) the path loss: $l\left(b, v, c_{v}\right)$, for each of its neighbor BS $b$.

Note that the measurement of signal power, interference and path loss $l(b, u, c)$ for each considered channel from either its own or neighboring base stations can be retrieved by the user terminal from the measurement of RSCP (received signal code power) and/or RSSI (received signal strength indication).

By the above information, each BS $b_{u}$ is able to compute $\mathcal{E}_{u}(\cdot)$. Notice that the neighbor cell communication takes place between base stations. So, there is no need to transmit the information via the wireless medium. We assume that this is supported by the backhaul network.

As aforementioned, the probability law $\pi_{u}(\mathbf{s})$ in (8) favors low energies. State transition is conducted to have the global energy minimization. It is known that the setting of temperature $T$ will impact the system's limiting distribution. It has to be chosen by taking a tradeoff between the convergence time and the strict optimality of the limit distribution. For a fixed environment (i.e., user population, signal attenuation), if we decrease $T$ in a logarithmic scale, the network will be driven to a state of minimal energy, starting from any arbitrary state. Here, we follow this and set $T=1 / \ln (1+t)$, where $t$ is time. A concrete proof of the convergence to the state of minimal $\mathcal{E}$ can be done similarly to that of [7, pp. 311-313] based on the notion of weak ergodicity of Markov chains and is thus omitted. In Section IV, we will illustrate the convergence and present numerical studies.

\section{SimUlation AND COMPARISON}

A performance investigation of the proposed solution is conducted below. We implement the optimization with Gibbs sampler and compare its performance with today's 3GPP default operations [9] by simulation.

To start with, by the current standard and 3G implementations, base stations are usually configured with a nominal fixed transmission power such that the pilot signal can be received by terminals over the covered area. The DL transmit power is often the maximum allowable power as well for a better user reception and coverage. The pilot signal is broadcasted continuously to allow user equipments to perform channel measurements and appropriate tuning. In user association, the current practice consists in attaching a user to the BS received with the strongest signal strength. Note that this could lead to attaching users to a far macro cell BS which has a higher transmission power than that of a nearer small cell BS. This is in general sub-optimal. In channel allocation, we consider that the system follows a heuristic scheme that channels of a BS are assigned to its users simply in a round-robin fashion, i.e., sequentially, such that the numbers of users (i.e., the load) in each of its channels will be well balanced and almost equal.

In simulation, we consider that users are uniformly distributed in a geographical area of 1000 meters times 650 meters and we adopt the 3GPP-3GPP2 spatial channel model [12]. The distance dependent path loss is given by:

$$
l^{(\mathrm{dB})}(d)=-\left(30.18+26 \log _{10}(d)+X_{\sigma}^{(\mathrm{dB})}\right),
$$

where $d$ is the transmitter-receiver distance and $X_{\sigma}$ refers to log-normal shadowing with zero mean and standard deviation $4 \mathrm{~dB}$. Consider operating temperature 290 Kelvin and bandwidth $1 \mathrm{MHz}, N_{u}$ is equal to $4.0039 \times 10^{-15} \mathrm{~W}, \forall u$.

Here, we consider that there are two fixed macro cell BS and a number of small cell BS. The latter are randomly located in the geographical area. The maximum transmit power of macro and small cell $\mathrm{BS}$ are $40 \mathrm{~W}$ and $1 \mathrm{~W}$, respectively. We assume that $P_{\delta}=0.1 \mathrm{~W}$. In the simulation, we consider a simple system where $\alpha=1$ and each user only takes one channel.

\section{A. Numerical Example}

To begin with, we illustrate the effectiveness of the algorithm by some examples with randomly generated small cell BS and users, as shown in Fig. 3-4. To have readable graphical representation and comparison of the user association, channel allocation and transmission power before and after optimization, in these examples we consider that the path loss is simply distance dependent without log-normal shadowing. So, a user who is farther from a BS has a larger path loss due to the greater distance. A line connecting a BS and a user indicates the user association and its thickness represents the strength of the transmit power. In these examples, we consider that there are two orthogonal channels in each BS, which are represented by different colors and line styles. Results (see Fig. 3-4) show that the proposed solution significantly outperforms the bydefault configuration in both system throughput (in $\mathrm{b} / \mathrm{s} / \mathrm{Hz}$ ) and power utilization efficiency (in $\mathrm{b} / \mathrm{s} / \mathrm{Hz} / \mathrm{W}$ ). Note that the latter has been improved by several orders of magnitude (also because our representation of the default operation has no power control mechanism). Notice that due to a lack of space, we have to omit the plots of algorithm convergence of the above examples. However, we observed that the algorithm usually converges within a few hundreds iterations and is hence practical.

\section{B. Average Performance}

Secondly, we compare the performance of the proposed optimization with the default operation, with a fixed number of $32 \mathrm{BS}$ (including the two macro BS) but with different numbers of users (denoted by $M$ ), i.e., different user densities, and different numbers of orthogonal channels (denoted by $K$ ). Users and small cells are randomly generated in the geographical area. For each $(M, K)$, data of 500 different topologies are obtained and the performance metrics are then averaged out. Table I shows the result, where the joint optimization has 


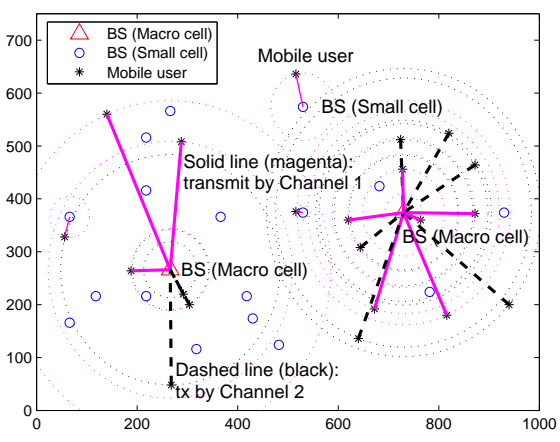

(a) Example 1: i) $8.7 \mathrm{~b} / \mathrm{s} / \mathrm{Hz}$, ii) $0.012 \mathrm{~b} / \mathrm{s} / \mathrm{Hz} / \mathrm{W}$

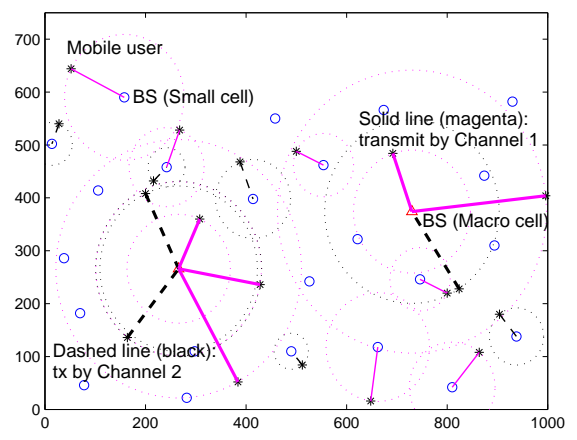

(b) Example 2: i) $35 \mathrm{~b} / \mathrm{s} / \mathrm{Hz}$, ii) $0.106 \mathrm{~b} / \mathrm{s} / \mathrm{Hz} / \mathrm{W}$

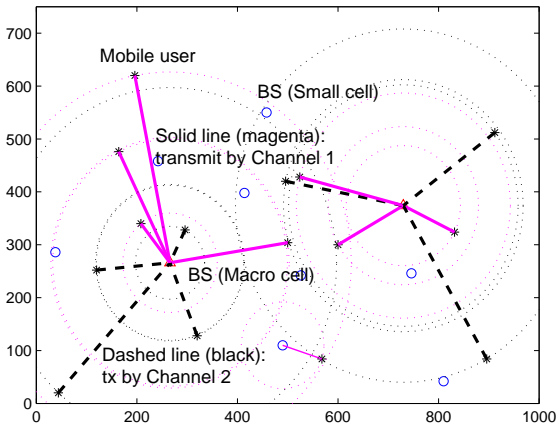

(c) Example 3: i) $7.5 \mathrm{~b} / \mathrm{s} / \mathrm{Hz}$, ii) $0.009 \mathrm{~b} / \mathrm{s} / \mathrm{Hz} / \mathrm{W}$

Fig. 3. Network before optimization. (a) Example 1: users are concentrated and fewer than BS. (b) Example 2: users are distributed and fewer than BS. (c) Example 3: more users than BS. Performance: i) system throughput, and ii) power efficiency. Two orthogonal channels: solid-magenta vs. dashed-black lines.

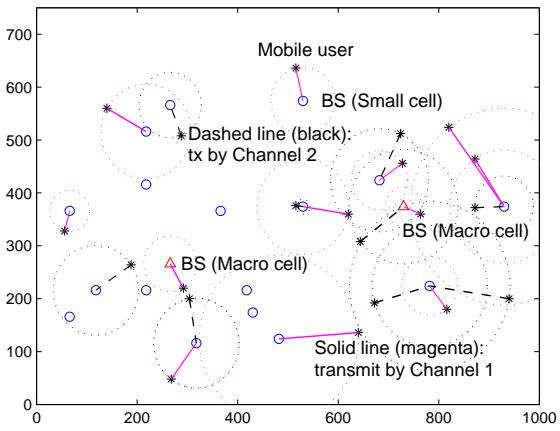

(a) Example 1: i) $43.5 \mathrm{~b} / \mathrm{s} / \mathrm{Hz}$, ii) $3.45 \mathrm{~b} / \mathrm{s} / \mathrm{Hz} / \mathrm{W}$

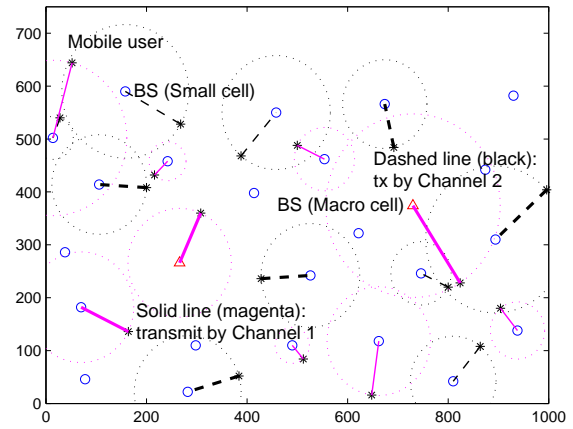

(b) Example 2: i) $75 \mathrm{~b} / \mathrm{s} / \mathrm{Hz}$, ii) $4.41 \mathrm{~b} / \mathrm{s} / \mathrm{Hz} / \mathrm{W}$

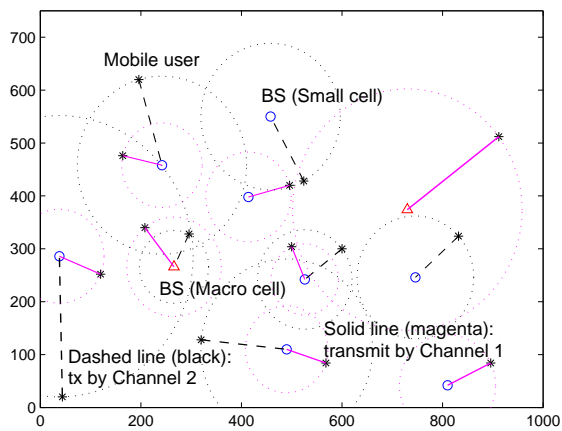

(c) Example 3: i) $32 \mathrm{~b} / \mathrm{s} / \mathrm{Hz}$, ii) $2.13 \mathrm{~b} / \mathrm{s} / \mathrm{Hz} / \mathrm{W}$

Fig. 4. Network after proposed joint optimization. Both the system throughput (b/s/Hz) and power utilization efficiency (b/s/Hz/W) are significantly improved.

substantially enhanced the system throughput and also power efficiency. It is observed that given the same $M / K$ ratio, the spectrum utilization efficiency after optimization also increases due to a higher degree of resource allocation flexibility (as $K$ increases), which is realized by the joint optimization. Note that the reflected scalability of the optimization is favorable when considering practical systems, e.g., in 3G HSDPA (HighSpeed Downlink Packet Access) and LTE, a high number of users is typical of dense network situations and a high number of resources is typical of data oriented networks.

\section{TABLE I}

USER AVERAGE THROUGHPUT: B/s/Hz, POWER EFFICIENCY: B/S/Hz/W

\begin{tabular}{c|c|c|c}
\hline & Default oper. & After optimiz. & Gain (times) \\
\hline \hline$M=32, K=1$ & $0.245,0.0143$ & $1.216,1.937$ & $4.96,135$ \\
\hline$M=64, K=2$ & $0.312,0.0186$ & $1.583,2.685$ & $5.07,144$ \\
\hline$M=96, K=3$ & $0.356,0.0210$ & $1.829,3.149$ & $5.14,150$ \\
\hline
\end{tabular}

\section{CONCLUSION}

In this paper, we identify the problem of radio resource allocation in heterogeneous cellular networks composed of macro and small cells with the unpredictability of cell and user patterns. To solve the problem completely, we propose a joint optimization of channel selection, user association and power control. Based on Gibbs sampler, the proposed solution is implementable in a fully distributed manner and nevertheless achieves minimal system-wide potential delay. Its operational simplicity is especially favorable for the target of network autonomic management and self-optimization. Simulation results have shown its effectiveness in both spectrum and energy utilization efficiencies. It has a clear potential in future macro and small cell mixed self-organizing networks.

\section{REFERENCES}

[1] C. Comaniciu, N. B. Mandayam, and H. V. Poor, "Radio resource management for green wireless networks," in IEEE VTC, 2009, pp. 1-5.

[2] V. Chandrasekhar, J. Andrews, and A. Gatherer, "Femtocell networks: a survey," IEEE Commun. Mag., vol. 46, no. 9, pp. 59-67, Sep. 2008.

[3] Next Generation Mobile Networks (NGMN). http://www.ngmn.org.

[4] L. C. Schmelz, J. L. van den Berg, R. Litjens, K. Zetterberg, M. Amirijoo, K. Spaey, I. Balan, N. Scully, and S. Stefanski, "Self-organisation in wireless networks - use cases and their interrelations," Wireless World Res. Forum Meeting 22, pp. 1-5, May 2009.

[5] 3GPP-LTE (Long Term Evolution). http://www.3gpp.org.

[6] I. Broustis, K. Papagiannaki, S. V. Krishnamurthy, M. Faloutsos, and V. Mhatre, "MDG: measurement-driven guidelines for 802.11 WLAN design," in MobiCom, 2007, pp. 254-265.

[7] P. Brémaud, Markov Chains: Gibbs Fields, Monte Carlo Simulation, and Queues. Springer Verlag, 1999.

[8] C. S. Chen and F. Baccelli, "Self-optimization in mobile cellular networks: power control and user association," in IEEE ICC, May 2010, pp. $1-6$.

[9] 3GPP TS 36.331, "Evolved universal terrestrial radio access radio resource control: Protocol specification," Tech. Spec. v9.2.0, 2010.

[10] L. Massoulié and J. Roberts, "Bandwidth sharing: objectives and algorithms," IEEE/ACM Trans. Netw., vol. 10, no. 3, pp. 320-328, 2002.

[11] B. Kauffmann, F. Baccelli, A. Chaintreau, V. Mhatre, K. Papagiannaki, and C. Diot, "Measurement-based self organization of interfering 802.11 wireless access networks," in IEEE INFOCOM, 2007, pp. 1451-1459.

[12] IEEE 802.20 Working Group on Mobile Broadband Wireless Access, "Channel models document," 3GPP-3GPP2 Tech. Rep., 2007. 